\title{
CYCLIC FATIGUE EFFECT IN PARTICULATE CERAMIC COMPOSITES
}

\author{
M. Lugovy ${ }^{1,2}$, V. Slyunyayev ${ }^{1}$, N. Orlovskaya ${ }^{2}{ }^{*}$, M. Reece $^{3}$, T. Graule ${ }^{4}$, J. Kuebler $^{4}$ \\ ${ }^{1}$ Institute for Problems of Materials Science, 3 Krzhizhanivskii Str., 03142 Kyiv, Ukraine \\ ${ }^{2}$ University of Central Florida, Orlando, 4000 Central Florida Blvd., Orlando, FL 32816, USA \\ ${ }^{3}$ Queen Mary University of London, School of Engineering and Material Science, London, UK \\ ${ }^{4}$ Empa, Swiss Federal Laboratories for Materials Science and Technology, Laboratory for \\ High Performance Ceramics, Ueberlandstrasse 129, 8600 Duebendorf, Switzerland \\ * Corresponding Author
}

\begin{abstract}
A new model is presented that provides an improved understanding of the time dependent fatigue behavior of two phase brittle particulate ceramic composites under static and cyclic loading conditions. The proposed model takes into consideration cyclic fatigue effects, which are responsible for the accelerated fatigue crack propagation in the cyclic loading as compared to the static loading. It also takes into account the effect of both thermal residual stresses and bridging stresses acting in the composite during time dependent crack propagation. Experimental results for the fatigue behavior of $\mathrm{ZrB}_{2}-45 \mathrm{vol} \% \mathrm{SiC}$ ceramic composite were used as a case study to valid the proposed model. The model gives insight both into the time dependent mechanical behavior of ceramic composites and, at the same time, allows determination of important structural parameter, such as, size of the bridging zone in the material under cycling loading.
\end{abstract}

Keywords: ceramics, fatigue, cyclic effect, bridging

\section{Introduction}

In many practical applications the lifetime and time dependent mechanical behavior of ceramic composites is determined by fatigue failure [1-13]. A material can experience both static and cyclic fatigue, where during the static fatigue the applied stress remains constant, while during 
cyclic loading the applied stress changes periodically from a minimum to a maximum value as a function of time. The lifetime of materials, such as porcelain and glass [14], is much shorter under static fatigue conditions in comparison with the lifetime of the same material tested under cyclic loading, when the maximum applied stress during cyclic experiment is equal to the stress applied during static fatigue test [15]. This is because in materials where crack growth is dominated by stress corrosion effects, during the cyclic fatigue the cracks experience lower average stresses in comparison with constant static stress.

However, for many ceramics, such as magnesia-partially-stabilized zirconia (Mg-PSZ) [16], $\mathrm{Ce}-\mathrm{TZP} / \mathrm{Al}_{2} \mathrm{O}_{3}$ composites [17], yttria-partially-stabilized zirconia (Y-TZP) [18, 19], silicon nitride $\left(\mathrm{Si}_{3} \mathrm{~N}_{4}\right)$ [20], alumina $\left(\mathrm{Al}_{2} \mathrm{O}_{3}\right)$ [21] and $\mathrm{Al}_{2} \mathrm{O}_{3}-\mathrm{SiC}$ whisker ceramic composite [22], the lifetime under cyclic fatigue loading was reported to be much shorter than the lifetime of the same material under static fatigue loading when that the maximum stress during cyclic testing is the same as a during static loading $[15,23]$. The decrease in lifetime under cyclic loading was explained by the degradation of the crack bridging elements under cyclic loading, leading to a reduction of their toughening effect (reduced closure force) [21, 23-25] (Fig. 1). As a crack propagates, new bridging elements are produced by the crack and the distance between them and crack tip increases. The bridging elements experience wear under the cyclic movement of crack surfaces, which decreases the friction forces and therefore bridging stress. The further the distance of the bridging element from crack tip, the higher is the wear rate due to the longer time of wear and the increasing crack opening displacement.

\section{Fett and Munz model}

While experimental and modeling work of the static and cyclic fatigue behavior of ceramic materials have been made in the past $[1-13,21,26]$, there have only been a few studies that have attempted to completely describe cyclic fatigue [24, 27]. An empirical model was proposed by Fett and Munz [24] based on a simple expression for average crack growth rate: 
$\mathrm{v}=A\left(K_{\max }-K_{b r}\right)^{n}$

where $\mathrm{v}$ is the average crack growth rate, $A$ and $n$ are the fatigue parameters, $K_{\max }$ is the maximum applied stress intensity factor in cyclic loading or constant applied stress intensity factor in static loading, $K_{b r}$ is the additional stress intensity factor due to bridging. In this model it was determined that the wear of bridging elements is responsible for a decrease of the bridging stress intensity factor $K_{b r}$ during cyclic loading, while during static loading $K_{b r}$ remains constant. It was proposed that at lower crack growth rates there was greater degradation of the bridging elements, leading to a lower bridging stress intensity factor $K_{b r}$ and shorter lifetime of the ceramic composite. A schematic plot of the dependence of the cyclic and static fatigue crack growth rate as a function of $K_{\max }$ is shown in Fig. 2. Above a critical value $\mathrm{v}=\mathrm{v}_{0}$ no degradation of bridging elements will occur and the $\mathrm{v}-K_{\max }$ dependences of cyclic and static fatigue coincide, thus no cyclic effect exists, as determined in the model in [24].

The Fett and Munz's model [24] did not discriminate between crack growth rates under static and cyclic fatigues for crack growth rates higher than $\mathrm{v}_{0}$ (Fig. 2). As presented by the model, the crack growth rates are identical, which is incorrect, as the average stress intensity factor for cyclic loading is always lower in comparison with the static loading if maximum applied stress intensity factor for cyclic loading is equal to applied stress intensity factor for static loading. Thus, in the absence of degradation of bridging elements, the crack growth rates will always be lower under cyclic loading in comparison with static loading for the case when the maximum cyclic and static loads are identical (Fig. 2). Another shortcoming of the model is an assumption that for the crack growth rates higher than $\mathrm{v}_{0}$ there is no no degradation of the bridging elements, thus no wear, is anticipated. However, while the wear will decrease as the crack growth rate increases, there is no physical reason to completely eliminate wear factor; it introduces a misconception about the real behavior of bridging stress intensity factor as a function of crack growth rate. In addition, the bridging stress intensity factor $K_{b r}$ in Eq. (1) is only taken into account in the model when the 
bridging stresses decrease the crack tip stress intensity factor. However, the friction implies that the bridging stress acts always against the change in crack surface displacements, therefore bridging will decrease crack tip intensity factor only when load is increasing during cyclic loading. For the portion of cyclic loading when the load is decreasing, the bridging will increase crack tip stress intensity factor, which is not taken into account in the model [24]. The above assumes that there is no elastic component to the bridging stress. The elastic ligaments, studied in [25], which always produce a closure component of the bridging stress, are not considered in the present work.

\section{Dauskardt model}

Another model, which accounts for cyclic fatigue effects involving wear of bridging elements in ceramics, was proposed by Dauskardt in [27]. The model used a differential equation for the calculation of residual stress $\sigma_{R}(x)$ acting in a bridging element of ceramic material upon crack propagation during loading:

$$
\frac{d \sigma_{R}}{d x}=\alpha_{W} k \frac{f}{\mathrm{v}} U(x) \sigma_{R}(x),
$$

where $x$ is the distance of bridging element from crack tip, $\alpha_{W}$ is the wear rate of the bridging element, $\mathrm{v}$ is the average crack growth rate, $f$ is the frequency of cyclic loading, $k$ is the proportionality constant which is a function of both the elastic properties and thermal expansion of both the bridging element itself and the surrounding material, $U(x)$ is the function which depends on crack opening displacement at the point with coordinate $x$. The model is not empirical, as in the previous case, as the decrease of $K_{b r}$ was estimated by calculating the decrease of $\sigma_{R}(x)$ and the corresponding decrease of the bridging stress. While the model provided realistic estimates for fatigue crack growth rates in ceramic materials when the cyclic effect is present, the model was applicable for single phase ceramics. Also, only cracks with periodic complete closing during cyclic loading, where the crack surface displacements are equal to zero, are considered. However, the crack does not close completely, especially when $K_{b r}$ is large, and the model does not account for 
such situations. In addition, in the model the crack tip stress intensity factor is assumed to be equal to an intrinsic fracture toughness of ceramics, corresponding to when no crack bridging effect is present. However, such a condition corresponds to catastrophic instantaneous fracture and is not applicable for time dependent fatigue behavior.

Therefore, the goal of the present study is to develop an improved and expanded model of fatigue failure of ceramic materials built on the previous models. The proposed model is capable of predicting time dependent fatigue behavior and describing the cyclic effect associated with degradation of crack bridging with a better precision.

\section{The proposed model}

\subsection{The description, assumptions and improvements}

The modified model proposed in this work is built on the instantaneous crack growth rate equation [15]:

$$
\mathrm{v}(t)=A_{s}\left(\frac{K_{t i p}(t)}{K_{1 c}}\right)^{n},
$$

where $\mathrm{v}(t)$ is the instantaneous crack growth rate, $A_{s}$ is a static fatigue parameter, $K_{1 c}$ is the fracture toughness of the material, and $K_{t i p}(t)$ is an instantaneous crack tip stress intensity factor as a function of time. Then $\mathrm{v}$, the average crack growth rate per cycle, can be calculated using the instantaneous crack growth rate $\mathrm{v}(t)(3)$ :

$$
\mathrm{v}=\frac{1}{T} \int_{0}^{T} \mathrm{v}(t) d t=\frac{A_{s}}{T K_{1 c}^{n}} \int_{0}^{T}\left[K_{\text {tip }}(t)\right]^{n} d t,
$$

where $T=1 / f$ is the period of cyclic loading, $f$ is the frequency of cyclic loading.

A few assumptions are made in order to further enhance the proposed model. First, it is proposed that the instantaneous crack tip stress intensity factor is equal to $K_{t i p}(t)=K_{\max }-K_{b r}$ with a time independent instantaneous crack growth rate in the case of static loading. For cyclic loading two cases are considered. In the first case, for the part of the cycle when the load increases, the 
friction forces will oppose to the crack opening, then $K_{t i p}(t)=K_{a}(t)-K_{b r}$, where $K_{a}(t)$ is the time dependent applied stress intensity factor. For the other portion of the cycle, when the load decreases and the friction forces oppose crack closure, then $K_{t i p}(t)=K_{a}(t)+K_{b r}$. The bridging stress intensity factor $K_{b r}$ is calculated using a bridging stress distribution along the moving crack, where the bridging stress itself is determined by friction forces acting at the interface between bridging elements and surrounding matrix. Finally, in the proposed model, the instantaneous crack growth rate $\mathrm{v}(t)$ is averaged over a full cycle to obtain the average crack growth rate. Such averaging of $\mathrm{v}(t)$ over the full loading cycle provides separate crack growth rate values for static and cyclic fatigue above the $\mathrm{v}_{0}$ value, unlike in the previous model [24] where both crack growth rates coincide (Fig. 2). In addition to the improvement of the model for discrimination of the static and cyclic fatigue crack growth rates, an attempt was made to model the wear of bridging elements. It is proposed that for the calculation of friction forces and wear of bridging elements both maximum and minimum crack opening displacements corresponding to maximum and minimum crack tip stress intensity factors of loading cycle should be taken into account. This was not done in [24], where only an empirical equation was used to account for wear and friction and, thus, no wear occurred for the crack growth rates higher than $\mathrm{v}_{0}$. Our model was also expanded to the case of a two-phase composite, where the different elastic properties and thermal expansion coefficients of the bridging elements and surrounding matrix were taken into account for calculation of residual stresses in bridging elements. Unlike in the previous two models [24, 27], the proposed model allows the calculation of an average crack growth rate $\mathrm{v}$ for cyclic fatigue which can be compared with experimentally obtained values. The detailed explanation of $K_{t i p}(t)$ and its constituents are provided below.

\subsection{Determination of the instantaneous crack tip stress intensity factor $K_{\text {tip }}(t)$}


The instantaneous crack tip stress intensity factor $K_{t i p}(t)$ can be determined taking into account friction and the resulting stress intensity factor $K_{b r}$ due to bridging for the increasing or decreasing loads parts of the cycle using the following equation

$$
K_{t i p}(t)=\left\{\begin{array}{lllll}
K^{-}(t), & 0 \leq t<T / 4, & K^{-}(t) \geq 0, & K_{\max }^{-}>K_{\min }^{+}, & K^{-}(t) \geq K_{\min }^{+} \\
K^{+}(t), & T / 4 \leq t<3 T / 4, & K^{+}(t) \geq 0, & K_{\max }^{-}>K_{\min }^{+}, & K^{+}(t) \leq K_{\max }^{-} \\
K^{-}(t), & 3 T / 4 \leq t \leq T, & K^{-}(t) \geq 0, & K_{\max }^{-}>K_{\min }^{+}, & K^{-}(t) \geq K_{\min }^{+} \\
K_{\min }^{+}, & 0 \leq t<T / 4, & K_{\min }^{+} \geq 0, & K_{\max }^{-}>K_{\min }^{+}, & K^{-}(t)<K_{\min }^{+} \\
K_{\max }^{-}, & T / 4 \leq t<3 T / 4, & K_{\max }^{-} \geq 0, & K_{\max }^{-}>K_{\min }^{+}, & K^{+}(t)>K_{\max }^{-} \\
K_{\min }^{+}, & 3 T / 4 \leq t \leq T, & K_{\min }^{+} \geq 0, & K_{\max }^{-}>K_{\min }^{+}, & K^{-}(t)<K_{\min }^{+} \\
0, & 0 \leq t<T / 4, & K^{-}(t)<0, & K_{\max }^{-}>K_{\min }^{+}, & K^{-}(t) \geq K_{\min }^{+} \\
0, & T / 4 \leq t<3 T / 4, & K^{+}(t)<0, & K_{\max }^{-}>K_{\min }^{+}, & K^{+}(t) \leq K_{\max }^{-} \\
0, & 3 T / 4 \leq t \leq T, & K^{-}(t)<0, & K_{\max }^{-}>K_{\min }^{+}, & K^{-}(t) \geq K_{\min }^{+} \\
0, & 0 \leq t<T / 4, & K_{\min }^{+}<0, & K_{\max }^{-}>K_{\min }^{+}, & K^{-}(t)<K_{\min }^{+} \\
0, & T / 4 \leq t<3 T / 4, & K_{\max }^{-}<0, & K_{\max }^{-}>K_{\min }^{+}, & K^{+}(t)>K_{\max }^{-} \\
0, & 3 T / 4 \leq t \leq T, & K_{\min }^{+}<0, & K_{\max }^{-}>K_{\min }^{+}, & K^{-}(t)<K_{\min }^{+} \\
K_{\max }^{-}, & 0 \leq t \leq T, & K_{\max }^{-} \geq 0, & K_{\max }^{-} \leq K_{\min }^{+} & \\
0, & 0 \leq t \leq T, & K_{\max }^{-}<0, & K_{\max }^{-} \leq K_{\min }^{+} &
\end{array}\right.
$$

where $K^{-}(t)=K_{a}(t)-K_{b r}$ and $K^{+}(t)=K_{a}(t)+K_{b r}$ are stress intensity factors where the additional stress intensity factor due to bridging either diminish or enhance the applied stress intensity factor, $K_{\max }^{-}=K_{\max }-K_{b r}, K_{\min }^{+}=K_{\min }+K_{b r}, K_{\min }$ is the minimum applied stress intensity factor during cyclic loading, $K_{\max }$ is the maximum applied stress intensity factor during cyclic loading or constant applied stress intensity factor in static loading (the same one as in Eq. (1)), $K_{a}(t)$ is time dependent applied stress intensity factor.

Based on the proposed model, the three most characteristic types of $K_{t i p}(t)$ behavior for a sinusoidal applied stress intensity factor are:

$$
K_{a}(t)=K_{\max }\left[\frac{1+R}{2}+\frac{1-R}{2} \sin \frac{2 \pi t}{T}\right],
$$

where $R=K_{\min } / K_{\max }$, as illustrated in Fig. 3. The first characteristic type of $K_{t i p}(t)$ behavior is considered when all of the cycling is under tension-tension loading conditions. While the second type is when there is both tension and compression loading during a single cycle. The third example 
is the unusual case when $K_{b r}$ is so large that the change in applied stress intensity factor between $K_{\min }$ and $K_{\max }$ is not large enough to overcome the frictional forces and the crack opening displacement is frozen and remains constant during cycling. As can be seen in Fig. 3, in all three cases (a, c, and e) the $K_{a}(t)$, as well as $K^{-}(t)$ diminished or $K^{+}(t)$ enhanced applied stress intensity factors vary sinusoidally while reaching their maximum and minimum values simultaneously at the maximum and minimum applied stress [15]. The lower $K^{-}(t)$ and upper $K^{+}(t)$ sinusoids account for the bridging effects occurring due to friction between bridging elements and crack edges (Fig. 3) during crack opening or closure for the cases when load is either increasing or decreasing, respectively. While $K_{a}(t), K^{-}(t)$, and $K^{+}(t)$ change their values sinusoidally, the instantaneous crack tip intensity factor $K_{t i p}(t)$ will be affected drastically depending on the following factors: a) if $K_{\min }^{+}>0$ where all applied cyclic loading is in tension (Fig. 3a), or b) if $K_{\min }^{+} \leq 0$ where both tensile and compressive loading occur upon cycling (Fig. 3c) or c) for the case when the $K_{b r}$ contribution is quite large resulting in the rare case that $K_{\max }^{-} \leq K_{\min }^{+}$(Fig. 3e). In all three examples, the bold lines represent the dependence of $K_{t i p}(t)$ as a function of time, where the shadowed areas below $K_{\text {tip }}(t)$ are used to calculate the average crack growth rate $\mathrm{v}$ during cyclic loading.

For the case when all of the cyclic loading occurs under tension, the $K_{t i p}(t)$ values either coincide with $K^{-}(t)$ upon increasing the applied tensile stress (points $1 \rightarrow 2$ on $K$ - $t$ diagram) or coincide with $K^{+}(t)$ when the applied tensile stress is decreasing upon unloading in a single cycle (points $3 \rightarrow 4$ on $K$ - $t$ diagram) (Fig. 3a). When the applied cyclic stress increases (points $1 \rightarrow 2$ on $K$ - $t$ diagram), the crack opening displacement (COD) increases too, as shown schematically in Fig. $3 b$. When the applied cyclic stress decreases (points $3 \rightarrow 4$ on $K$ - $t$ diagram), the COD decreases (Fig. 3b). However, because of bridging frictional forces, the change of the applied stress from loading to unloading will not cause an immediate decrease in COD, but will prevent an immediate 
initiation of crack closure, which will result in the constant $K_{t i p}(t)=K_{\max }^{-}$(points $2 \rightarrow 3$ on $K-t$ diagram) (Fig. 3a). The same situation will repeat when unloading changes to the next loading cycle and the COD remain constants (points $4 \rightarrow 5$ on $K$ - $t$ diagram) (Fig. 3a, b) until the threshold frictional forces are overcome and the crack edges start opening again as the tensile load increases.

When both tension and compression are present during cycling and $K_{\min }^{+} \leq 0$, the COD from point 1 to 2 will not increase because $K_{t i p}(t)$ remains equal to zero and constant despite the increased $K_{a}(t)$ (Fig. 3c). However, when the applied crack stress intensity factor $K_{a}(t)$ exceeds $K_{b r}$, the crack starts to open from point 2 to 3 (Fig. 3c) and the COD increases (Fig. 3d). When $K_{a}(t)$ decreases during the unloading part of the cycle, $K_{t i p}(t)$ will decrease from point 4 to 5 (Fig. 3c) leading to almost complete closure of the crack, and the COD becomes equal to zero again, as at the beginning loading (Fig. 3d). However, at the very beginning of the unloading cycle, similar to the first case when all of the cycling occurs under tension (Fig. 3a), the presence of frictional forces will retard the crack closure (Fig. $3 \mathrm{~d}$ ) resulting in the constant $K_{t i p}(t)=K_{\max }^{-}$(points $3 \rightarrow 4$ on $K$ - $t$ diagram) (Fig. 3c). As crack opening displacements can adopt only positive values or be equal to zero when the crack is closed, negative $K_{t i p}(t)$ has no physical meaning, as it is directly proportional to COD. Therefore, a case where $K_{t i p}(t)$ becomes negative is not considered here.

When $K_{b r}$ is rather large and $K_{\max }^{-} \leq K_{\min }^{+}, K_{b r}$ the COD is frozen if the change in applied stress intensity factor between $K_{\max }$ and $K_{\min }$ is insufficient to overcome the frictional forces (Fig. 3e). In this case $K_{t i p}(t)=K_{\max }^{-}$if $K_{\max }^{-}>0$, and $K_{t i p}(t)$ becomes constant and independent of time leading to no degradation of bridging elements with no cyclic effect present (Fig. 3f).

Thus the developed model shows that the dependence of $K_{\text {tip }}(t)$ does not follow the sinusoidal dependence of applied stress intensity factor, but has its own behavior where both $K_{\min }$ 
and $K_{\max }$ values are not reached, thus allowing the average crack growth rate v to be calculated in a more accurate way.

\subsection{Determination of bridging stress intensity factor $K_{b r}$}

One of the important constituents of $K_{t i p}(t)$ is the stress intensity factor $K_{b r}$ acting due to the friction of the bridging elements during opening and closure of the moving crack during cycling. A schematic of the bridging elements within the edges of the moving crack, as well as definitions used in the equations for descriptions of the $K_{b r}$ stress intensity factor are shown in Fig. 4. The general expression for bridging stress intensity factor of a semicircular surface crack are presented below [15]

$$
K_{b r}=\int_{0}^{c} h(c, r) \sigma_{b r}(r) d r
$$

where $r$ is the radial coordinate, $c$ is the radius of semicircular surface crack, which defines the size of the fracture origin in the material, $\sigma_{b r}(r)$ is the distribution of bridging stress along $r$, and

$$
h(c, r)=\frac{\psi r}{c^{1 / 2}\left(c^{2}-r^{2}\right)^{1 / 2}},
$$

is the weighted function with $\psi$ being the geometrical factor (for a semicircular surface crack we can use $\psi=1.264[28])$.

The frictional forces are dependent on normal stresses at the interface. The normal stress at the interface between bridging element and matrix is equal to thermal residual stress $\sigma_{R}(r)$ in the bridging element. The following equation is utilized for the calculation of the bridging stress [27, 29]:

$\sigma_{b r}(r)=-2 \mu f_{b} \sigma_{R}(r)\left(d_{\text {out }}-2 u(r)\right) / d_{\text {in }}$,

where $\mu$ is the friction coefficient, $f_{b}=\frac{\pi d_{i n}^{2}}{4 l^{2}}, d_{i n}$ is the diameter of the bridging elements in crack plane, $d_{\text {out }}$ is the height of the frictional surface of the bridging element in the $y$-direction, $l$ is the 
average distance between bridging elements, and $u(r)$ is the half crack opening displacement at the crack edges. Such dependence $\sigma_{b r}(r)$ on $\sigma_{R}(r)$ can be used for the cases when the normal stress at the interface between bridging element and matrix is equal to the thermal residual stress $\sigma_{R}(r)$ in bridging element.

The $u(r)$ for a semicircular surface crack is calculated using the following equation developed by $[28,30]$ :

$u(r)=\frac{\psi K_{t i p}(t)\left(1-v_{1}^{2}\right)\left(c^{2}-r^{2}\right)^{1 / 2}}{E_{1} c^{1 / 2}}$,

where $E_{1}$ and $v_{1}$ are the Young's modulus and Poisson's ratio of matrix, respectively. The matrix material in the proposed two phase material's model is considered as a simple mixture of two phases. Thus, the $E_{1}$ and $v_{1}$ elastic properties can be either calculated by the rule of mixture using the $E$ and $v$ values of the pure phases, or it could be directly measured for the two phase composite material of interest.

The Eqs (7) and (9) demonstrate that $K_{b r}$ exhibits a linear dependence on $K_{t i p}(t)$ because $u(r)$ is a linear function of $K_{t i p}$ (10). Therefore, after substituting Eq. (10) into (9), and then Eq. (9) into (7), Eq. (7) can be rewritten as

$$
K_{b r}=a K_{t i p}(t)+b,
$$

where $a$ and $b$ are constants and are equal to

$$
a=\frac{4 \psi \mu f_{b}\left(1-v_{2}^{2}\right)}{d_{i n} E_{2} c^{1 / 2}} \int_{0}^{c}\left(c^{2}-r^{2}\right)^{1 / 2} h(c, r) \sigma_{R}(r) d r,
$$

and

$$
b=-\frac{2 \mu f_{b} d_{\text {out }}}{d_{\text {in }}} \int_{0}^{c} h(c, r) \sigma_{R}(r) d r
$$

$K_{t i p}(t)$ is difficult to determine, however, $K_{t i p}(t)$ has a linear dependence on the applied stress intensity factor $K_{a}(t)$, thus $K_{b r}$ will be also a linear function of $K_{a}(t)$. Therefore, Eq. (11) can be 
further rewritten to express $K_{b r}$ as a linear function of $K_{a}(t)$ for the two different cases of increasing and decreasing applied load.

For the first case, when the applied load increases in the time interval $0 \leq t<T / 4$ and $3 T / 4 \leq t \leq T$ during cycling (Fig. 3), the $K_{t i p}(t)=K_{a}(t)-K_{b r}$ and

$K_{b r}=a^{-} K_{a}(t)+b^{-}$,

where

$a^{-}=\frac{a}{1+a}$,

$b^{-}=\frac{b}{1+a}$.

For the case when the applied load decreases in the cycle for $T / 4 \leq t<3 T / 4, K_{t i p}(t)=K_{a}(t)+K_{b r}$ and

$K_{b r}=a^{+} K_{a}(t)+b^{+}$,

where

$a^{+}=\frac{a}{1-a}$,

$b^{+}=\frac{b}{1-a}$.

As $K_{a}(t)$ can be expressed using the applied stress $\sigma_{a}(t)$ by the equation $K_{a}(t)=\psi \sigma_{a}(t) \sqrt{c}$, then we can state that $K_{a}(t)$ can be directly estimated by knowing the applied cyclic stress, therefore $K_{b r}$ can be also directly calculated.

\subsection{Determination of thermal residual stress $\sigma_{R}(r)$ in the bridging element}

For a two phase composite material thermal residual stress $\sigma_{R}(r)$ can be estimated in the bridging element with a radial coordinate $r$, where the strengthening phase is considered as the bridging elements and the mixture of two phases is considered as a matrix, in which bridging 
elements operate. When a crack propagates further into the two phase matrix, the bridging element of the second phase become captured by the moving crack and has a tendency to debond away from the matrix, while before debonding it is rigidly connected to the other grains in the two phase matrix material $[27,31,32]$. In the first approximation, after debonding, the equiaxial bridging element can be considered as a combination of a cylindrical central part which is in a frictional contact with a matrix and a free open end (Fig. 4). For such a bridging element, the thermal residual stress $\sigma_{R}(r)$ can be calculated using the following equation for cylinders which have a frictional contact surface due to thermal mismatch [33]:

$$
\sigma_{R}(r)=\sigma_{R 0}+\frac{2 E_{1} E_{2}}{d_{\text {in }}\left[\left(1-v_{2}\right) E_{1}+\left(1+v_{1}\right) E_{2}\right]} h(r),
$$

where $E_{1}$ and $E_{2}$ are Young's moduli and $v_{1}$ and $v_{2}$ are the Poisson's ratios of two phase matrix material and the phase producing the bridging elements, respectively, $h(r)$ is the linear wear representing a decrease in the thermal mismatch due to wear at the interface between the bridging elements and matrix phases. The $\sigma_{R 0}$ term represents the initial thermal residual stress existing in the two phase composite due to mismatch of the coefficients of thermal expansion of different phases, when no wear occurs. The $\sigma_{R 0}$ is expressed by the equation:

$$
\sigma_{R 0}=\frac{E_{1} E_{2} \Delta \alpha \Delta T}{\left(1-v_{2}\right) E_{1}+\left(1+v_{1}\right) E_{2}},
$$

where $\Delta T=T_{R}-T_{j}, T_{R}$ is an actual operational temperature, $T_{j}$ is a "joining" temperature, where both two phases of the material become rigidly bonded to one another upon cooling from high processing temperature, $\Delta \alpha=\alpha_{1}-\alpha_{2}$, where $\alpha_{1}$ and $\alpha_{2}$ are coefficients of thermal expansion of the whole two phase matrix material and the phase constituting the bridging elements, respectively.

The linear wear $h(r)$ in the right part of Eq. (20) is a complex function of $r$ due to different durations of wear for the elements having different radial coordinates. As it is a complex function it is difficult or simply impossible to determine $h(r)$ directly, either by direct experiment or by other means. Thus, Eq. (20) cannot be used directly to determine the thermal residual stress in the 
bridging element. Therefore, the following approach for developing of the function relating thermal residual stresses $\sigma_{R}$ to the radial coordinate $r$ of the bridging element was adopted and further developed for a two phase material [27]. Firstly, Eq. (20) was differentiated with respect to $x$, where $x=c-r$ (Fig. 4a), and the following equation was obtained

$$
\frac{d \sigma_{R}}{d x}=\frac{2 E_{1} E_{2}}{d_{\text {in }}\left[\left(1-v_{1}\right) E_{2}+\left(1+v_{2}\right) E_{1}\right]} \frac{d h}{d x} .
$$

Secondly, if we assume that the crack extension per cycle is equal to

$$
\Delta x=\mathrm{v} / f
$$

and it is small enough that $\sigma_{R}(x)$ can be considered as a constant over this extension, then we can replace $\frac{d h}{d x}$ with the following

$$
\frac{d h}{d x} \rightarrow \frac{\Delta h}{\Delta x}
$$

where $\Delta h=\Delta h(x)$ is a linear wear per cycle, which is possible for the vast majority of fatigue crack growth rates and loading frequencies of interest. Then, thirdly, the total volume of material removed from the bridging element-matrix interface per one cycle can be expressed as

$$
\Delta V_{W}(x)=A_{f} \Delta h(x)
$$

where $A_{f}=\pi d_{\text {in }} d_{\text {out }} / 2$ is the sliding contact area between the bridging element and the matrix. Eq. (23a) is applicable for the case of one half of the bridging element embedded on one side of the crack at position $x$ in the bridging zone (Fig. 4c). By combining Eqs. (22a) and (23a) we obtain

$$
\frac{\Delta h}{\Delta x}=\frac{\Delta V_{W} f}{A_{f} \mathrm{~V}} .
$$

In the Eq. (24) the $\Delta V_{W}(x)$ term has to be defined in order to have all terms for the equation defining thermal residual stress $\sigma_{R}(x)$ in the bridging element available. It is not a simple task, and the following approach was used to define the total volume of the material removed per cycle. It is known that the wear at the interface between the bridging element and the matrix can be also 
expressed by a simple relationship. The volume of the material $V_{W}$ removed due to wear is given by [27]:

$V_{W}=\alpha_{W} \sigma_{N} s F$,

where $\alpha_{W}$ is the wear rate, $\sigma_{N}$ is the normal stress at the interface which is equal to $\sigma_{R}(x), s$ is the sliding distance for any point of the contact area $F$. It is important to understand that the wear rate $\alpha_{W}$ is presented as a constant here for the simplification. The real wear processes of bridging element are much more complex than those described by Eq. (25), however, the direct measurements and quantifications of them are simply impossible. Thus Eq. (25), which includes a constant wear rate $\alpha_{W}$, provides a reasonable approximation for the wear at the interface of the bridging element and the matrix of a two phase composite [27].

The Eq. (25) contains the sliding distance $s$ for any point of the contact area between the matrix and the bridging element, and it is obvious that the sliding distance is different for points with different coordinate $y$ on the interface (Fig. 4c). Because of this, Eq. (25) can only be applied to the frictional surface of an infinitesimal annular portion of the bridging element with coordinate $y$ (Fig. 4c). Using Eq. (25) this infinitesimal volume $d V_{W}$ of the material worn away by friction at the annular portion of the bridging element during single loading cycle is presented by the equation: $d V_{W}(x, y)=-\alpha_{W} \cdot \sigma_{R}(x) \cdot \Delta s(x, y) \cdot d A_{f}$

where $d A_{f}=\pi d_{i n} d y$. The sliding distance $\Delta s(x, y)$ per loading cycle along $y$-direction of the annular element in the Eq. (26) is

$\Delta s(x, y)=\left\{\begin{array}{cc}0, & 0 \leq y<u_{\text {min }}(x) \\ 2\left[y-u_{\text {min }}(x)\right], & u_{\text {min }}(x) \leq y<u_{\text {max }}(x), \\ 2\left[u_{\text {max }}(x)-u_{\text {min }}(x)\right], & u_{\text {max }}(x) \leq y\end{array}\right.$

where $u_{\min }(x)$ and $u_{\max }(x)$ are, respectively, minimum and maximum half crack opening displacements of the crack at the location of the bridging element. $u_{\text {min }}(x)$ and $u_{\max }(x)$ can be determined using Eq. (10) with the minimum and maximum $K_{t i p}(t)$ values in the cycle, 
respectively. Then, the total volume of material $\Delta V_{W}$ removed per cycle from the bridging elementmatrix interface for a one half of the bridging elements embedded on one side of the crack at position $x$ in the bridging zone can be found by integrating the Eq. (26) with respect to $y$ from $y=0$ to $y=d_{\text {out }} / 2$ :

$\Delta V_{W}=-\alpha_{W} \cdot \sigma_{R}(x) \cdot \pi d_{i n} \cdot\left[u_{\max }(x)-u_{\min }(x)\right] \cdot\left[d_{\text {out }}-u_{\max }(x)-u_{\min }(x)\right]$.

The Eq. (28), as expressed, will take into account the possibility of incomplete closure of the crack during the loading cycle because it includes $u_{\text {min }}(x)$, which can be non-zero. Finally, using Eqs. (28), (23), and (24), Eq. (22) can be transformed into a differential equation which expresses thermal residual stress $\sigma_{R}(x)$ in the bridging element as:

$$
\frac{d \sigma_{R}}{d x}=\frac{4 E_{1} E_{2} \alpha_{W} f}{d_{\text {in }} d_{\text {out }} \mathrm{v}\left[\left(1-v_{1}\right) E_{2}+\left(1+v_{2}\right) E_{1}\right]} \cdot \sigma_{R}(x) \cdot\left[u_{\text {max }}(x)-u_{\text {min }}(x)\right] \cdot\left[d_{\text {out }}-u_{\text {max }}(x)-u_{\text {min }}(x)\right] .
$$

The Eq. (29) can be solved numerically using Runge-Kutta schemes [34].

\subsection{The required input parameters of the proposed model}

As one can see from the proposed model description, in order to calculate an average crack growth rate $\mathrm{v}$ of a semicircular surface crack, with radius $c$, many initial parameters have to be known. Besides values of the external maximum applied stress intensity factor $K_{\max }$, the parameter $R$, and the frequency $f$ of the cycling, the elastic properties $E_{1}, E_{2}, v_{1}$, and $v_{2}$ as well as the coefficients of thermal expansion $\alpha_{1}$ and $\alpha_{2}$ of the two phase matrix material and the bridging elements, respectively, have to be known. Besides, the so called "joining temperature" $T_{j}$ along with the fracture toughness $K_{1 c}$ of the composites should be also available as input parameters to the model. In addition, the average size of the bridging element $d_{i n}$ and the average distance between bridging elements $l$ should be also estimated. A more complicated problem is to estimate the friction coefficient $\mu$, the effective size of frictional surface in the $y$-direction $d_{\text {out }}$, the wear rate $\alpha_{W}$, the static fatigue parameter $A_{s}$, and the fatigue parameters $n$. While many parameters, 
such as elastic properties, coefficients of thermal expansion, size of the bridging elements as well as the distance between them can be directly measured, $A_{s}$ and $n$ fatigue parameters can only be determined experimentally. The coefficient of friction $\mu$ values can only be fitted in the proposed model, similar to the fitting performed in [31].

\section{Verification of the proposed model $-\mathrm{ZrB}_{2}-45$ vol\% $\mathrm{SiC}$ ceramic composite as a case study}

In order to validate the proposed cyclic fatigue model for the two phase brittle ceramic composite, $\mathrm{ZrB}_{2}-45 \mathrm{vol} \% \mathrm{SiC}$ ceramic composite was chosen as a model material as it is a well characterized material from previous research [35-42]. The required input parameters for the model, mentioned in section 4.5 of the paper, are listed in Table 1 . The joining temperature $T_{j}$ was chosen to be equal to the sintering temperature $\left(2120{ }^{\circ} \mathrm{C}\right)$ of $\mathrm{ZrB}_{2}-45 \mathrm{vol} \% \mathrm{SiC}$ ceramic composite prepared by hot pressing [35]. The $\alpha_{1}$ and $\alpha_{2}$ coefficients of thermal expansion (CTE) were determined to be equal to $\alpha_{1}=6.4 \cdot 10^{-6} \mathrm{~K}^{-1}$ for $\mathrm{ZrB}_{2}-45 \mathrm{vol} \% \mathrm{SiC}$ and $\alpha_{2}=5.07 \cdot 10^{-6} \mathrm{~K}^{-1}$ for $\mathrm{SiC}$ [38]. The radius $c$ of the semicircular surface crack was estimated using the well-known $K_{1 c}=\psi \sigma_{c} \sqrt{c}$ expression, where $\sigma_{c}=688 \mathrm{MPa}$ was estimated from the strength data of $\mathrm{ZrB}_{2}-45 \mathrm{vol} \% \mathrm{SiC}$ ceramic composite [37]. The $\sigma_{c}$ value is slightly different from the average strength of the composite presented in [35] because the strength was calculated for fatigue tested specimens. The calculated value of $c=19 \mu \mathrm{m}$ is in a good agreement with the experimentally observed size of fracture origins when fracture initiated from the surface of ceramic composite during loading [35]. The static fatigue parameters $n$ and $A_{s}$ were obtained [37] taking into account the bridging stress intensity factor $K_{b r}=0.95$ $\mathrm{MPa} \cdot \mathrm{m}^{0.5}$. The average size of the bridging elements $d_{i n}=2 \pm 0.36 \mu \mathrm{m}$ and an average distance $l=4.5$ $\mu \mathrm{m}$ between the bridging elements were measured by microscopy analysis of the microstructure of $\mathrm{ZrB}_{2}-45 \mathrm{vol} \% \mathrm{SiC}$ ceramic composites. The microstructure of the composite and crack and bridging elements used in analysis are shown in Fig. 5; similar micrographs were used to analyze 100 bridging elements. 
To obtain the value of coefficient of friction $\mu$ and the effective size of the frictional surface in the $y$-direction $d_{\text {out }}$, Eqs. (7) and (9) were used. The value of thermal residual stress $\sigma_{R 0}$ in the SiC bridging elements used in Eqs. (7) and (9) to perform the fitting for estimation of $\mu$ and $d_{\text {out }}$ was calculated using Eq. (21) to be equal to $633 \mathrm{MPa}$. The $K_{b r}$, also used in these equations, is equal to $0.95 \mathrm{MPa} \cdot \mathrm{m}^{0.5}$ as was estimated in previous research on $\mathrm{ZrB}_{2}-45 \mathrm{vol} \% \mathrm{SiC}$ ceramic composite [37]. It is known that $\mu$ can be larger than 1 and have a limit of about 3 [31], while the effective size $d_{\text {out }}$ is smaller than $d_{\text {in }}$ and is within the range $0.05 d_{\text {in }} \leq d_{\text {out }} \leq 0.3 d_{\text {in }}$. Using these values, the best fit estimated the value for $\mu=3$ and $d_{\text {out }}=0.6 \mu \mathrm{m}$. The wear rate $\alpha_{W}=4 \cdot 10^{-6}$ $\mathrm{mm}^{3} / \mathrm{Nm}$ was estimated by fitting a theoretical dependence of $\mathrm{v}\left(K_{\max }\right)$ for cyclic fatigue with the corresponding experimental data (Fig. 6).

The theoretical and experimental dependences of $\lg \mathrm{v}-\lg K_{\max } / K_{1 \mathrm{c}}$ for static and cyclic fatigue of $\mathrm{ZrB}_{2}-45 \mathrm{vol} \% \mathrm{SiC}$ ceramic composite are shown in Fig. 6. The experimental measurements were obtained in [37]. The developed model provides a theoretical curve with a good fit to the experimental data for both static and cyclic fatigue. As can be seen in Fig. 6, the theoretical curve for cyclic fatigue intersects the theoretical curve for static fatigue at high crack growth rates, almost approaching the dashed line which represents the $\lg \mathrm{v}-\lg K_{\max } / K_{1 \mathrm{c}}$ dependence when the effect of cycling does not appear during cyclic fatigue testing, as it was reported for glass, porcelain, and other ceramic materials [14, 15]. The developed model clearly shows a decrease of wear of bridging elements and a decrease of cyclic effects with an increase of crack growth rate.

It is important to understand that using the proposed model the size of the bridging zone along the length of the moving crack can be estimated using the distributions of residual and bridging stresses along the crack length for different $K_{\max } / K_{1 \mathrm{c}}$ ratio. The calculated distributions of normalized residual stress $\sigma_{R} / \sigma_{R 0}$ and normalized bridging stress $\sigma_{b r} / \sigma_{b r 0}$, where $\sigma_{b r 0}=-2 \mu f_{b} \sigma_{R 0} d_{\text {out }} / d_{\text {in }}$, along a crack for four different $K_{\max } / K_{1 \mathrm{c}}$ ratios are shown in Fig. 7 . For the 
applied stress intensity factor $K_{\max }$ which is only slightly less than the measured fracture toughness of the composite, such that $K_{\max } / K_{1 \mathrm{c}}=0.99$, the degradation of residual and bridging stresses is quite small due to the rather high crack growth rates and, as a result, the small wear of the bridging elements during cycling (Fig. 7a). When the $K_{\max } / K_{1 \mathrm{c}}$ ratio decreases to 0.922 , the larger degradation of the residual and bridging stresses is predicted (Fig. 7b) as the decrease of the crack growth rates and increase in wear of the bridging elements are more pronounced in comparison with the previous case when $K_{\max } / K_{1 \mathrm{c}}=0.99$. Even further degradation of the residual and bridging stresses occur if the applied stress intensity factor $K_{\max }$ is further decreased such that the $K_{\max } / K_{1 \mathrm{c}}$ ratio becomes equal to 0.848 (Fig. $7 \mathrm{c}$ ). For $K_{\max } / K_{1 \mathrm{c}}=0.82$, the part of the crack with $0.7 \leq x / c \leq 1$ will bear no bridging stress (Fig. 7d); this information can be used to determine the end of the bridging zone, which can be located for the cases where the bridging stress is equal to zero. The size of bridging zone $l_{b}$ is normalized by the radius $c$ of the semicircular surface crack and dependence of $l_{b} / c$ on $K_{\max } / K_{1 \mathrm{c}}$ is calculated using the provided input parameters of the model in the Table 1. As one can see from Fig. 8, for of $K_{\max } / K_{1 \mathrm{c}}$ ratio of 0.82 , when the crack growth rate is quite high, the bridging zone size is constant and equal to the crack radius $c$, as the degradation of the bridging elements does not have sufficient time to decrease the bridging zone size during crack propagation. For smaller $K_{\max } / K_{1 \mathrm{c}}$ values, where the crack growth rate is lower, the size of the bridging zone decreases rapidly due to significant wear of the bridging elements. The bridging zone size approaches zero and the bridging zone itself almost disappears when $K_{\max } / K_{1 \mathrm{c}}$ becomes equal to 0.6.

Based on the developed model, the dependences of bridging stress intensity factor $K_{b r}$ as a function of the crack growth rate $\mathrm{v}$ for $\mathrm{ZrB}_{2}-45 \mathrm{vol} \% \mathrm{SiC}$ ceramic composite for static and cyclic fatigue tests are plotted in Fig. 9. As one can see from Fig. 9, the almost horizontal line presents the results for static testing; in the proposed model there is slight decrease of the bridging stress intensity factor with increasing crack growth rate. Such a decrease follows from the fact that the higher crack growth rate corresponds to a higher stress intensity factor $K_{\max }$ and larger crack opening displacement, which results in a smaller friction surface between bridging elements and 
matrix and as a result of this slightly lower bridging stresses. However, the decrease in $K_{b r}$ is small, so that the good agreement between the proposed model and model developed in [24] is still observed. The bridging stress intensity factor for static fatigue was assumed to be a constant and independent on the crack growth rate in the Fett and Munz model. The sloping dashed line shows $K_{b \mathrm{r}} / K_{1 \mathrm{c}}$ for cyclic testing as calculated using the model from [24]. This is a straight line in a log-log plot, and intersects the horizontal line at the point of some critical crack growth rate. However, the proposed model gives much more accurate results for the $\lg K_{b_{\mathrm{r}}} / K_{1 \mathrm{c}} \mathrm{vs} \lg \mathrm{v}$ dependence, as it shows an almost linear plot for the lower crack growth rates and provides a non-linear dependence with a saturation trend for the higher crack growth rates where the $K_{b \mathrm{r}} / K_{1 \mathrm{c}}$ ratio for cyclic tests approaches the values for static tests. For comparison, the calculations using Fett and Munz model [24] provide only a linear $\lg K_{b \mathrm{r}} / K_{1 \mathrm{c}} \mathrm{vs} \lg \mathrm{v}$ dependence, thus do not provide as accurate a description of the cyclic effect as it possible to obtain using the proposed model for two phase brittle ceramic composites.

\section{Conclusions}

The revised and expanded model for the static and cyclic fatigue behavior of two phase particulate brittle ceramic composites was developed based on the previous work of Fett and Munz [24] and Dauskardt [27]. The model describes the $\lg \mathrm{v}-\lg K_{\max } / K_{1 \mathrm{c}}$ dependence of a two phase brittle ceramic composite for static and cyclic fatigue. The $\lg \mathrm{v}-\lg K_{\max } / K_{1 \mathrm{c}}$ dependence of the model correlates well with the experimental data for the cases when cyclic effects are either present or absent (high crack growth rates). The proposed model takes into account both the effect of thermal residual stresses occurring due to mismatch of the coefficients of thermal expansion between the two ceramic phases and, also, the effect of the bridging stress due to so called "crack bridging" mechanism, where the bridging elements of the material experience wear due to frictional forces, thus facilitating the accelerated crack propagation and shortening lifetime of ceramics under cyclic fatigue conditions. The bridging stress, considered by the model, always acts against the 
change in the crack opening displacement when a frictional bridging mechanism is present. The model is also capable of predicting the decrease in wear of the bridging elements with increasing crack growth rate, while in the previous model the wear disappears completely and abruptly when the crack growth rate increases above a certain value [24]. Another advantage of the proposed model is that it takes into account the fact that a moving crack may not experience complete closure. Another advantage of the proposed model is that the size of the bridging zone can easily be determined by building and using the bridging stress distribution.

\section{Acknowledgement}

This work was supported by the National Science Foundation, the Division of Materials Research, project \# 0748364 in the Ceramics program. Dr. Slyunyayev's work at Empa was supported by Swiss National Science Foundation via the International Short Visit fellowship (Decision IZK0Z2_147715). Dr. Lugovy's work at Empa was supported by Swiss National Science Foundation via the International Short Visit fellowship (Decision IZK0Z2_134265). 


\section{References}

1. M. Reece, F. Guiu, Repeated indentation method for studying cyclic fatigue in ceramics, J. Am. Ceram. Soc. 73 (4) (1990) 1004-1013.

2. M. Reece, F. Guiu, Indentation fatigue of high-purity alumina in fluid environments, J. Am. Ceram. Soc. 74 (1) (1991) 148-154.

3. F. Guiu, M.J. Reece, D.A.J. Vaughan, Cyclic fatigue of ceramics, J. Mater. Sci. 26 (12) (1991) 3275-3286.

4. P. Hvizdos, M.J. Reece, R. Torrecillas, Fatigue behaviour of mullite studied by the indentation flexure method, J. Eur. Ceram. Soc. 21 (1) (2001) 53-61.

5. R.H. Dauskardt, W. Yu, R.O. Ritchie, Fatigue crack propagation in transformation-toughened zirconia ceramic, J. Am. Ceram. Soc. 70 (10) (1987) C-248-C-252.

6. F. Guiu, Cyclic fatigue of polycrystalline alumina in direct push-pull, J. Mater. Sci. 13 (6) (1978) 1357-1361.

7. L.S. Williams, Stress endurance of sintered alumina, Trans. Br. Ceram. Soc. 55 (5) (1956) 287312.

8. L.S. Williams, Fatigue and ceramics, in: W.W. Kriegel and H. Palmour, editors. Mechanical Properties of Engineering Ceramics. Interscience, New York, 1961.

9. B.K. Sarkar, T.G.T. Glinn, Fatigue behavior of high- $\mathrm{Al}_{2} \mathrm{O}_{3}$ ceramics, Trans. Br. Ceram. Soc. 69 (1970) 199-203.

10. D.A. Krohn, D.P.H. Hasselman, Static and cyclic fatigue behavior of a polycrystalline alumina, J. Am. Ceram. Soc. 55 (4) (1972) 208-211.

11. D. Lewis, R.W. Rice, Comparison of static, cyclic, and thermal-shock fatigue in ceramic composites, Ceram. Eng. Sci. Proc. 3 (9-10) (1982) 714-721.

12. H.N. Ko, Fatigue strength of sintered $\mathrm{Al}_{2} \mathrm{O}_{3}$ under rotary bending, J. Mater. Sci. Lett. 5 (4) (1986) 464-466. 
13. H.N. Ko, Fatigue strength of sintered $\mathrm{Si}_{3} \mathrm{~N}_{4}$ under rotary bending, J. Mater. Sci. Lett. 6 (2) (1987) 175-177.

14. A.G. Evans, E.R. Fuller, Crack prapagation in ceramic materials under cyclic loading conditions, Met. Trans. 5 (1) (1974) 27-33.

15. D. Munz, T. Fett, Ceramics: Mechanical Properties, Failure Behaviour, Materials Selection. Springer, Berlin, Heidelberg, New York, 1999.

16. A.A. Steffen, R.H. Dauskardt, R.O. Ritchie, Cyclic fatigue life and crack-growth behavior of microstructurally small cracks in magnesia-partially-stabilized zirconia ceramics, J. Am. Ceram. Soc. 74 (6) (1991) 1259-1268.

17. J.F. Tsai, C.S. Yu, D.K. Shetty, Fatigue crack propagation in ceria-partially-stabilized zirconia (Ce-TZP)-alumina composites, J. Am. Ceram. Soc. 73 (10) (1990) 2992-3001.

18. S.-Y. Liu, I-W. Chen, Fatigue of yttria-stabilized zirconia: I, Fatigue damage, fracture origins, and lifetime prediction, J. Am. Ceram. Soc. 74 (6) (1991) 1197-1205.

19. S.-Y. Liu, I-W. Chen, Fatigue of yttria-stabilized zirconia: II, Crack propagation, fatigue striations, and short-crack behavior, J. Am. Ceram. Soc. 74 (6) (1991) 1206-1216.

20. S. Horibe, Fatigue of silicon nitride ceramics under cyclic loading, J. Eur. Ceram. Soc. 6 (2) (1990) 89-95.

21. M.J. Reece, F. Guiu, M.F.R. Sammur, Cyclic fatigue crack propagation in alumina under direct tension-compression loading, J. Am. Ceram. Soc. 72 (2) (1989) 348-352.

22. R.H. Dauskardt, M.R. James, J.R. Porter, R.O. Ritchie, Cyclic fatigue-crack growth in a SiCwhisker-reinforced alumina ceramic composite: Long- and small-crack behavior, J. Am. Ceram. Soc. 75 (4) (1992) 759-771.

23. T. Fett, D. Munz, Differences between static and cyclic fatigue effects in alumina, J. Mater. Sci. Lett. 12 (5) (1993) 352-354.

24. T. Fett, D. Munz, A relation for cyclic crack growth in ceramics, J. Mater. Sci. Lett. 17 (4) (1998) 307-309. 
25. F. Guiu, M. Li, M.J. Reece, Role of crack-bridging ligaments in the cyclic fatigue behavior of alumina, J. Am. Ceram. Soc. 75 (11) (1992) 2976-2984.

26. S. Lathabai, J. Roedel, B.R. Lawn, Cyclic fatigue from frictional degradation at bridging grains in alumina, J. Am. Ceram. Soc. 74 (6) (1991) 1340-1348.

27. R.H. Dauskardt, A frictional-wear mechanism for fatigue-crack growth in grain bridging ceramics, Acta Metall. Mater. 41 (9) (1993) 2765-2781.

28. R.F. Cook, C.J. Fairbanks, B.R. Lawn, Y.-W. Mai, Crack resistance by interfacial bridging: Its role in determining strength characteristics, J. Mater. Res. 2 (3) (1987) 345-356.

29. T. Fett, Friction-induced bridging effects caused by the T-stress, Eng. Fract. Mech. 59 (5) (1998) 599-606.

30. I.N. Sneddon, The distribution of stress in the neighbourhood of a crack in an elastic solid, Proc. R. Soc. A 187 (1009) (1946) 229-260.

31. S.J. Bennison, B.R. Lawn, Role of interfacial grain-bridging sliding friction in the crackresistance and strength properties of nontransforming ceramics, Acta Metall. 37 (10) (1989) 26592671.

32. N.P. Padture, J.L. Runyan, S.J. Bennison, L.M. Braun, B.R. Lawn, Model for toughness curves in two-phase ceramics: II, Microstructural variables, J. Am. Ceram. Soc. 76 (9) (1993) 2241-2247.

33. S. Timoshenko, Strength of Materials. Part II: Advanced theory and Problems. D. Appleton \& Co, 1956.

34. G.A. Korn, T.M. Korn, Mathematical Handbook for Scientists and Engineers, McGraw-Hill, New York, San Francisco, Toronto, London, Sydney, 1968.

35. N. Orlovskaya, R. Stadelmann, M. Lugovy, V. Subbotin, G. Subhash, M. Neubert, C.G. Aneziris, T. Graule, J. Kuebler, Mechanical properties of $\mathrm{ZrB}_{2}-\mathrm{SiC}$ ceramic composites: room temperature instantaneous behaviour, Adv. Appl. Ceram. 112 (1) (2013) 9-16.

36. M. Lugovy, N. Orlovskaya, M. Neubert, C.G. Aneziris, T. Graule, J. Kuebler, Room temperature fatigue of $\mathrm{ZrB}_{2}-\mathrm{SiC}$ ceramic composites, Ceram. Int. 39 (8) (2013) 9187-9194. 
37. M. Lugovy, N. Orlovskaya, M. Neubert, C.G. Aneziris, T. Graule, J. Kuebler, Time dependent mechanical properties of $\mathrm{ZrB}_{2}-\mathrm{SiC}$ ceramic composites: room temperature fatigue parameters, Sci. Adv. Mater. 6 (4) (2014) 844-852.

38. M. Lugovy, V. Slyunyayev, N. Orlovskaya, E. Mitrentsis, C.G. Aneziris, T. Graule, J. Kuebler, Temperature dependence of elastic properties of $\mathrm{ZrB}_{2}-\mathrm{SiC}$ composites, Ceram. Int. 42 (2A) (2016) 2439-2445.

39. R. Stadelmann, M. Lugovy, N. Orlovskaya, P. Mchaffey, M. Radovic, V.M. Sglavo, S. Grasso, M.J. Reece, Mechanical properties and residual stresses in $\mathrm{ZrB}_{2}-\mathrm{SiC}$ spark plasma sintered ceramic composites, J. Eur. Ceram. Soc. 2015 (In Press).

40. R. Stadelmann, B. Hughes, N. Orlovskaya, S. Grasso, M.J. Reece, 2D Raman mapping and thermal residual stresses in $\mathrm{SiC}$ grains of $\mathrm{ZrB}_{2}-\mathrm{SiC}$ ceramic composites, Ceram. Int. 41 (10A) (2015) 13630-13637.

41. R. Stadelmann, B. Hughes, N. Orlovskaya, Guideline of mapping parameters for Raman mapping of $\mathrm{ZrB}_{2}-\mathrm{SiC}$ ceramic composites, Adv. Appl. Ceram. 115 (1) (2016) 21-28.

42. N. Fist, J. Dinan, R. Stadelmann, N. Orlovskaya, An in-situ three-point bending device for measurements of vibrational response of ceramics under stress by micro Raman spectroscopy, Adv. Appl. Ceram. 111 (7) (2012) 433-439. 
Table 1. Input parameters for the model

\begin{tabular}{|c|c|}
\hline Parameter & Value \\
\hline Frequency of cyclic tests, $f, \mathrm{~Hz}$ & 10 \\
\hline$R$ (for static test) & 1 \\
\hline$R$ (for cyclic test) & -1 \\
\hline Static fatigue parameter, $A_{s}, \mathrm{~m} / \mathrm{s}$ & 2.143 \\
\hline Static fatigue parameter, $n$ & 50.7 \\
\hline Semicircular surface crack radius, $c, \mu \mathrm{m}$ & 19 \\
\hline "Joining" temperature, $T_{j},{ }^{\circ} \mathrm{C}$ & 2120 \\
\hline Fracture toughness, $K_{1 c}, \mathrm{MPa} \cdot \mathrm{m}^{0.5}$ & 3.8 \\
\hline Diameter of bridging element in crack plane, $d_{i n}, \mu \mathrm{m}$ & 2 \\
\hline Effective size of frictional surface in $y$-direction, $d_{o u t}, \mu \mathrm{m}$ & 0.6 \\
\hline Average distance between bridging elements, $l, \mu \mathrm{m}$ & 4.5 \\
\hline Friction coefficient, $\mu$ & 3 \\
\hline Wear rate, $\alpha_{W}, \mathrm{~mm}^{3} / \mathrm{Nm}$ & $4 \cdot 10^{-6}$ \\
\hline Young's modulus of two phase matrix, $E_{1}, \mathrm{GPa}$ & 476 \\
\hline Poisson's ratio of two phase matrix, $v_{1}$ & 0.167 \\
\hline Coefficient of thermal expansion of two phase matrix, $\alpha_{1}, 10^{-6} \mathrm{~K}^{-1}$ & 6.4 \\
\hline Young's modulus of bridging element, $E_{2}, \mathrm{GPa}$ & 410 \\
\hline Poisson's ratio of bridging element, $v_{2}$ & 0.188 \\
\hline Coefficient of thermal expansion of bridging element, $\alpha_{2}, 10^{-6} \mathrm{~K}^{-1}$ & 5.07 \\
\hline
\end{tabular}




\section{Figure captions}

Fig. 1. Schematic of crack bridging.

Fig. 2. Schematic dependences $\lg v-\lg \mathrm{K}_{\max }$ for static and cyclic fatigue. $\mathrm{v}_{\mathrm{s}}$ is static fatigue crack growth rate; $\mathrm{v}_{\mathrm{c}}$ is cyclic fatigue crack growth rate.

Fig. 3. Instantaneous crack tip stress intensity factor for sinusoidal cyclic loading: a) $K_{t i p}^{\text {min }}>0$; b) $K_{\text {tip }}^{\min } \leq 0$; c) $K_{t i p}^{\max } \leq K_{t i p}^{\min }$. Bold line is instantaneous crack tip stress intensity factor. The middle sinusoids correspond to applied stress intensity factor. Shaded area shows integration area to calculate average crack growth rate for cyclic loading. The conditions of crack opening displacement $\delta$, as shown in $\mathrm{b}, \mathrm{d}$, and $\mathrm{f}$, in the characteristic points $1,2,3,4,5$ of the plots a, c, and d, respectively, are as follows: for the case (a) - $\delta_{1}<\delta_{2}=\delta_{3}>\delta_{4}=\delta_{5}$; for the case (c) $\delta_{1}=\delta_{2} \approx 0<\delta_{3}=\delta_{4}>\delta_{5} \approx 0$; for the case (e) $-\delta_{1}=\delta_{2}=\delta_{3}=\delta_{4}=\delta_{5}$.

Fig. 4. Schematic of crack and bridging elements in the model considered.

Fig. 5. Bridging elements and their characteristics in $\mathrm{ZrB}_{2}-45 \mathrm{vol} \% \mathrm{SiC}$ composite.

Fig. 6. Theoretical and experimental dependences $\lg \mathrm{v}-\lg \mathrm{K}_{\mathrm{max}} / \mathrm{K}_{1 \mathrm{c}}$ for static and cyclic fatigue of $\mathrm{ZrB}_{2}-45 \mathrm{vol} \% \mathrm{SiC}$ composite. Circles are the experimental data for cyclic fatigue. Squares are the experimental data for static fatigue. Dashed line corresponds to the theoretical dependence when cyclic effects do not occur.

Fig. 7. Dependences of residual stress and bridging stress for different $K_{\max } / K_{1 c}$ : a) $K_{\max } / K_{1 c}=0.99$;

b) $\mathrm{K}_{\max } / \mathrm{K}_{1 \mathrm{c}}=0.922$; c) $\mathrm{K}_{\max } / \mathrm{K}_{1 \mathrm{c}}=0.848$; d) $\mathrm{K}_{\max } / \mathrm{K}_{1 \mathrm{c}}=0.82$. Solid line corresponds to residual stress. Dashed line corresponds to bridging stress.

Fig. 8. Dependence of normalized bridging zone size on $\mathrm{K}_{\max } / \mathrm{K}_{1 \mathrm{c}}$ ratio.

Fig. 9. Dependence of bridging stress intensity factor on crack growth rate for $\mathrm{ZrB}_{2}-45 \mathrm{vol} \% \mathrm{SiC}$ composite. Dashed lines correspond to the model from [24]. 


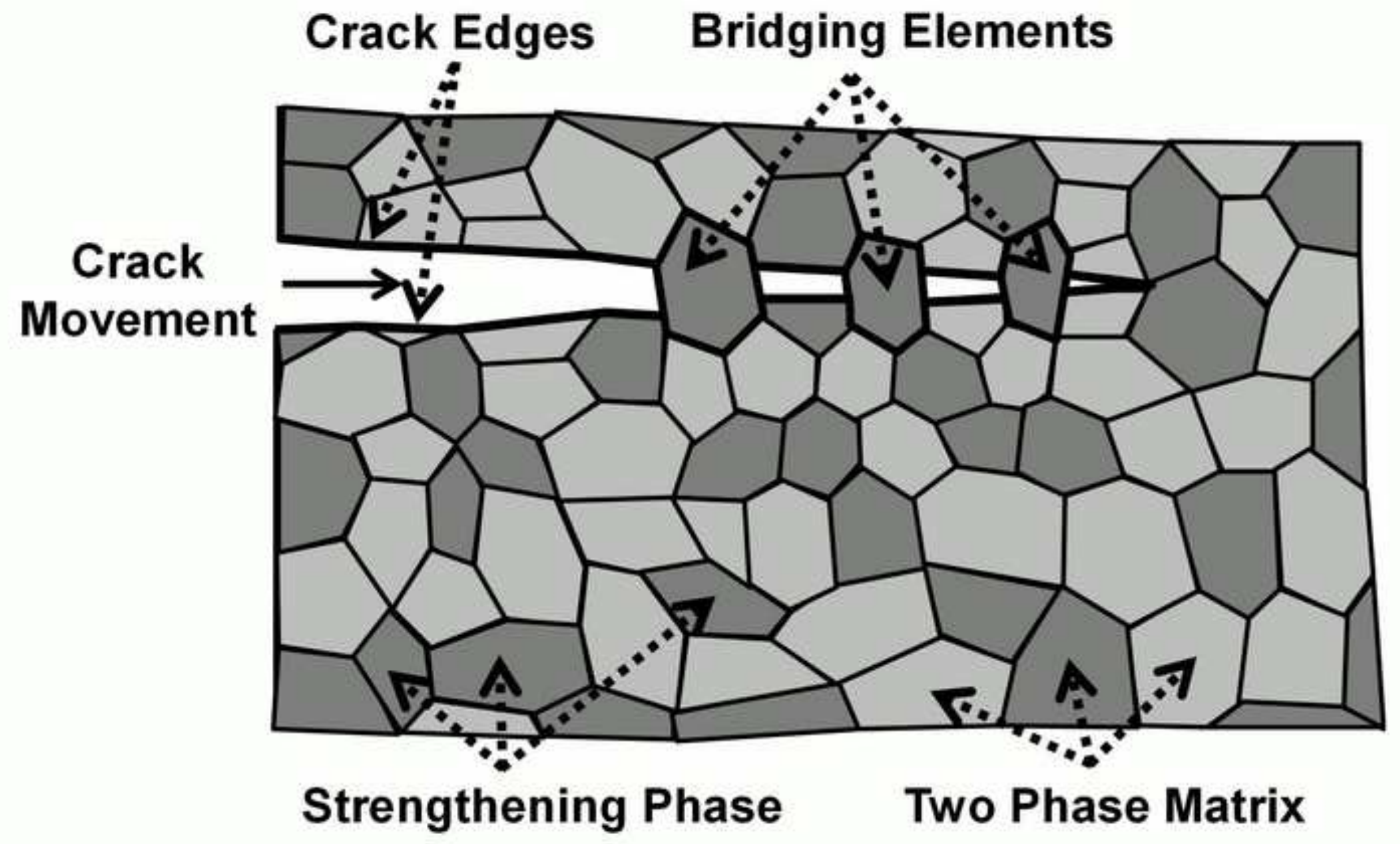




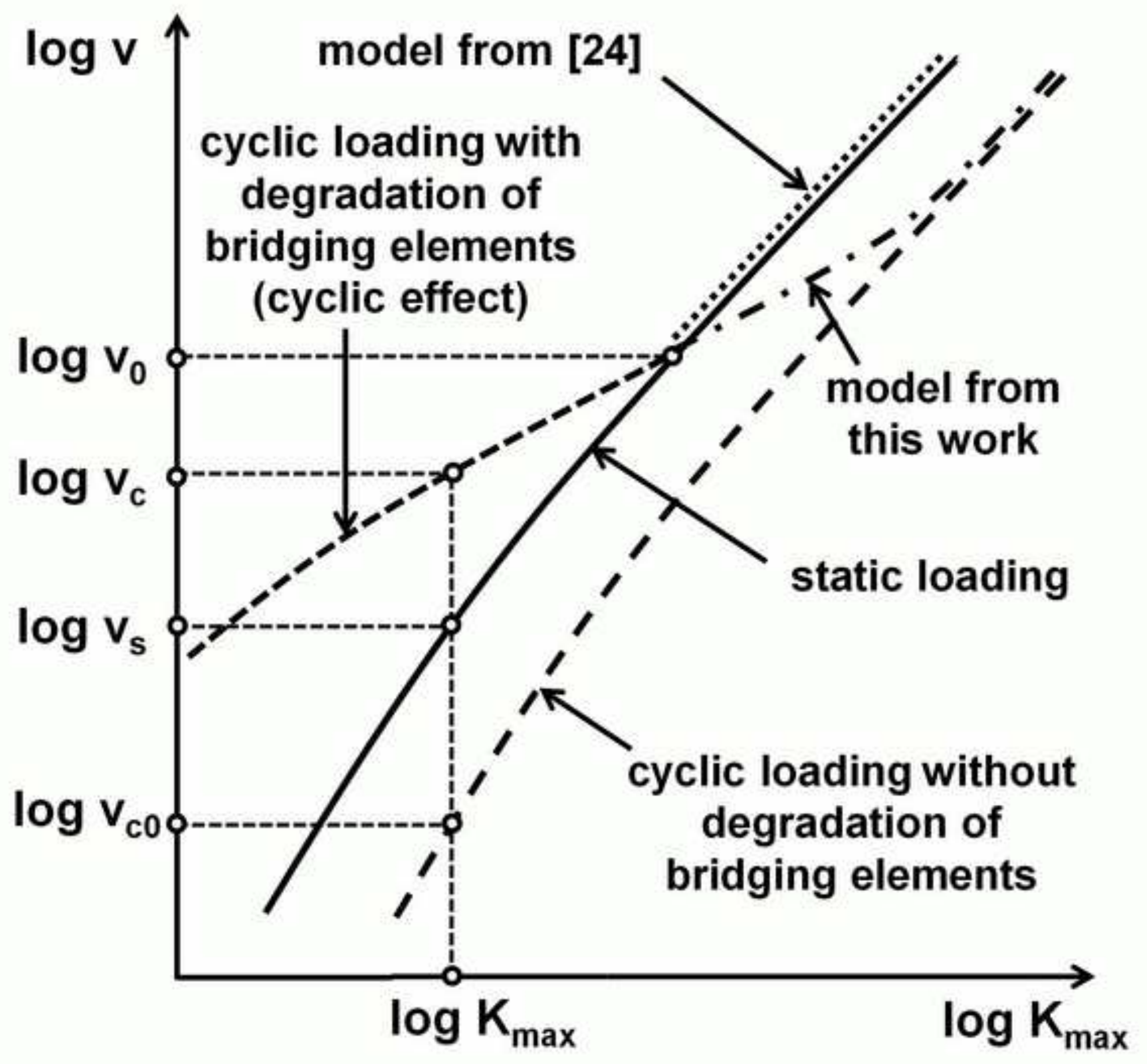




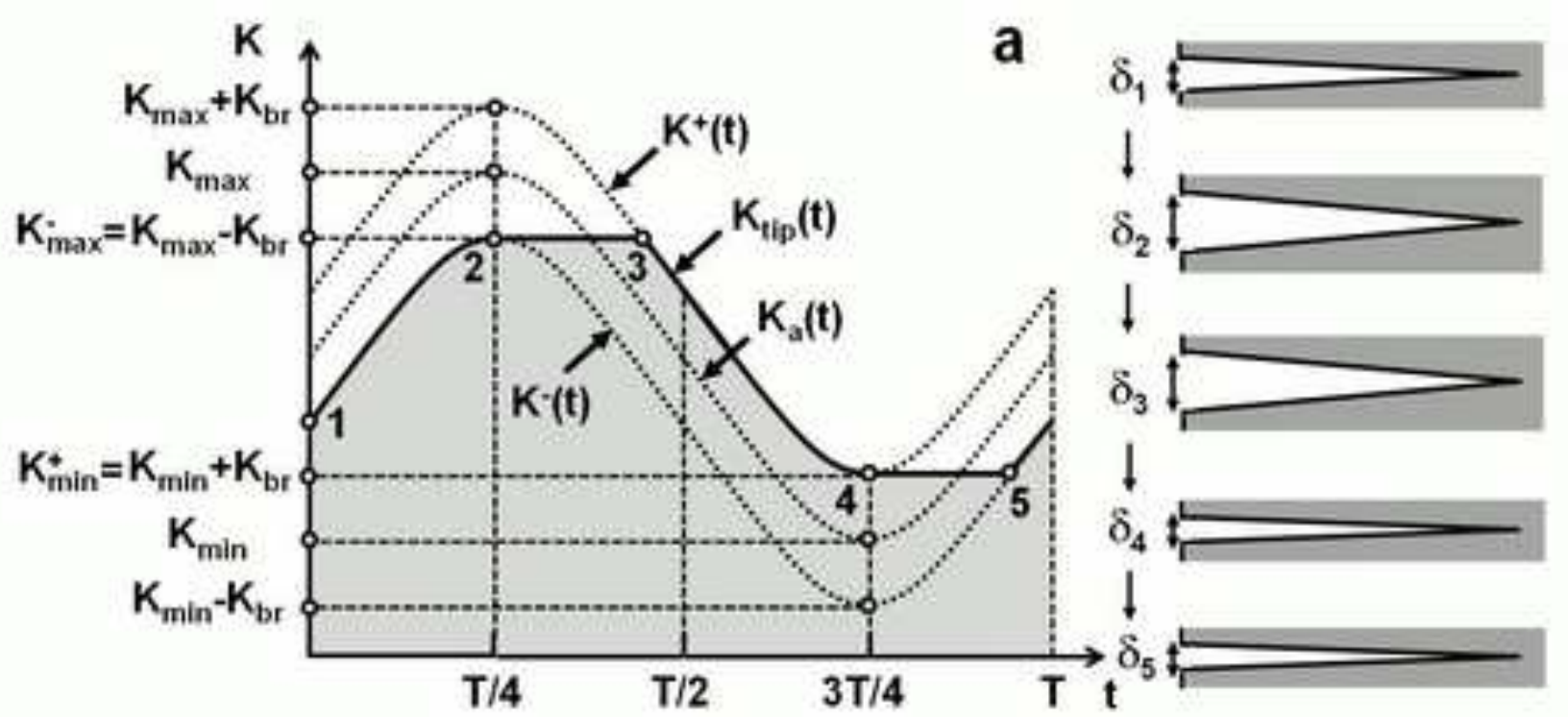

b
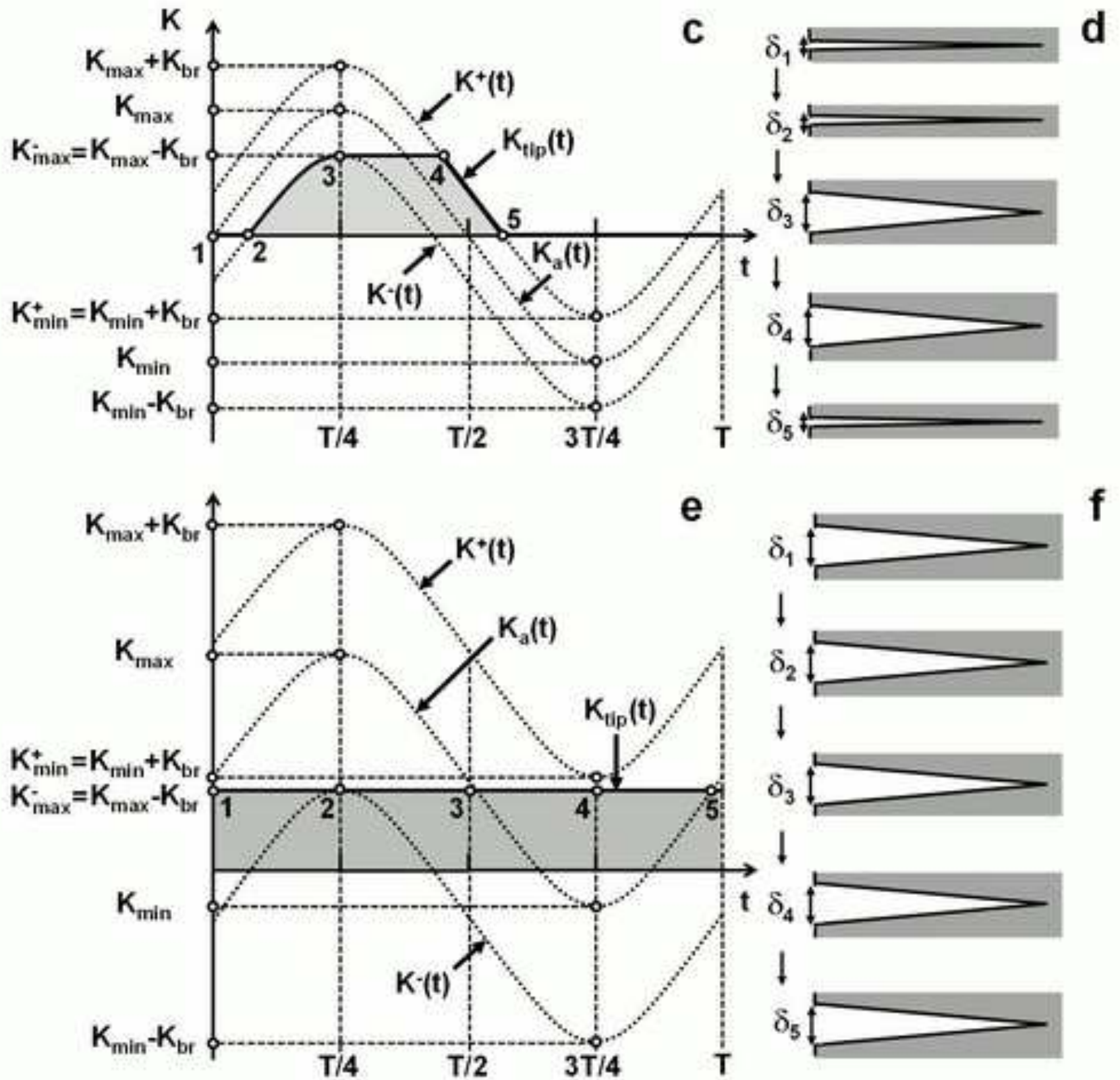


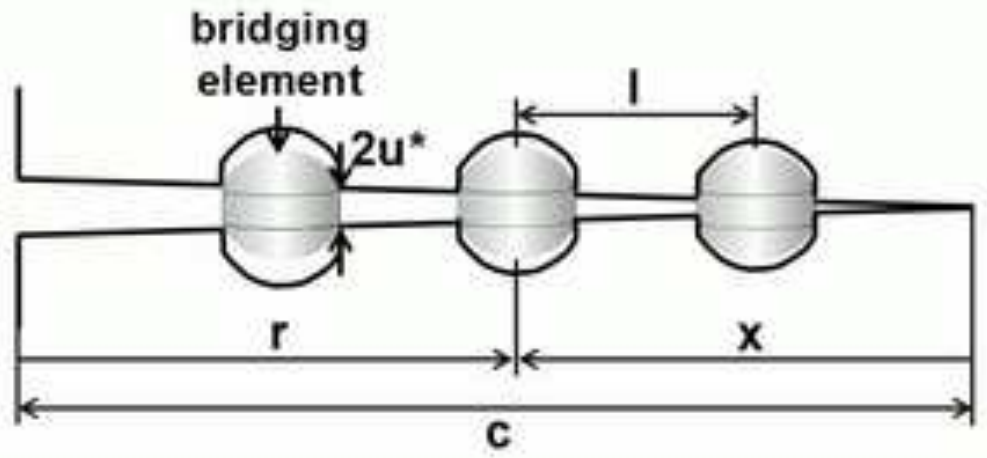

a

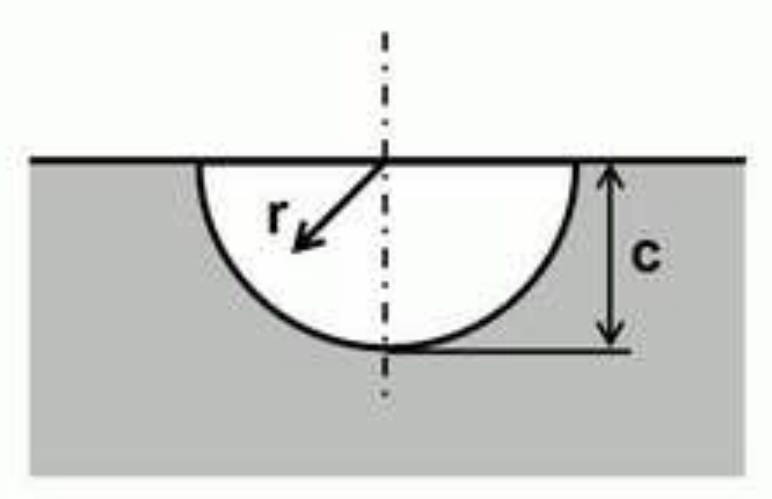

b

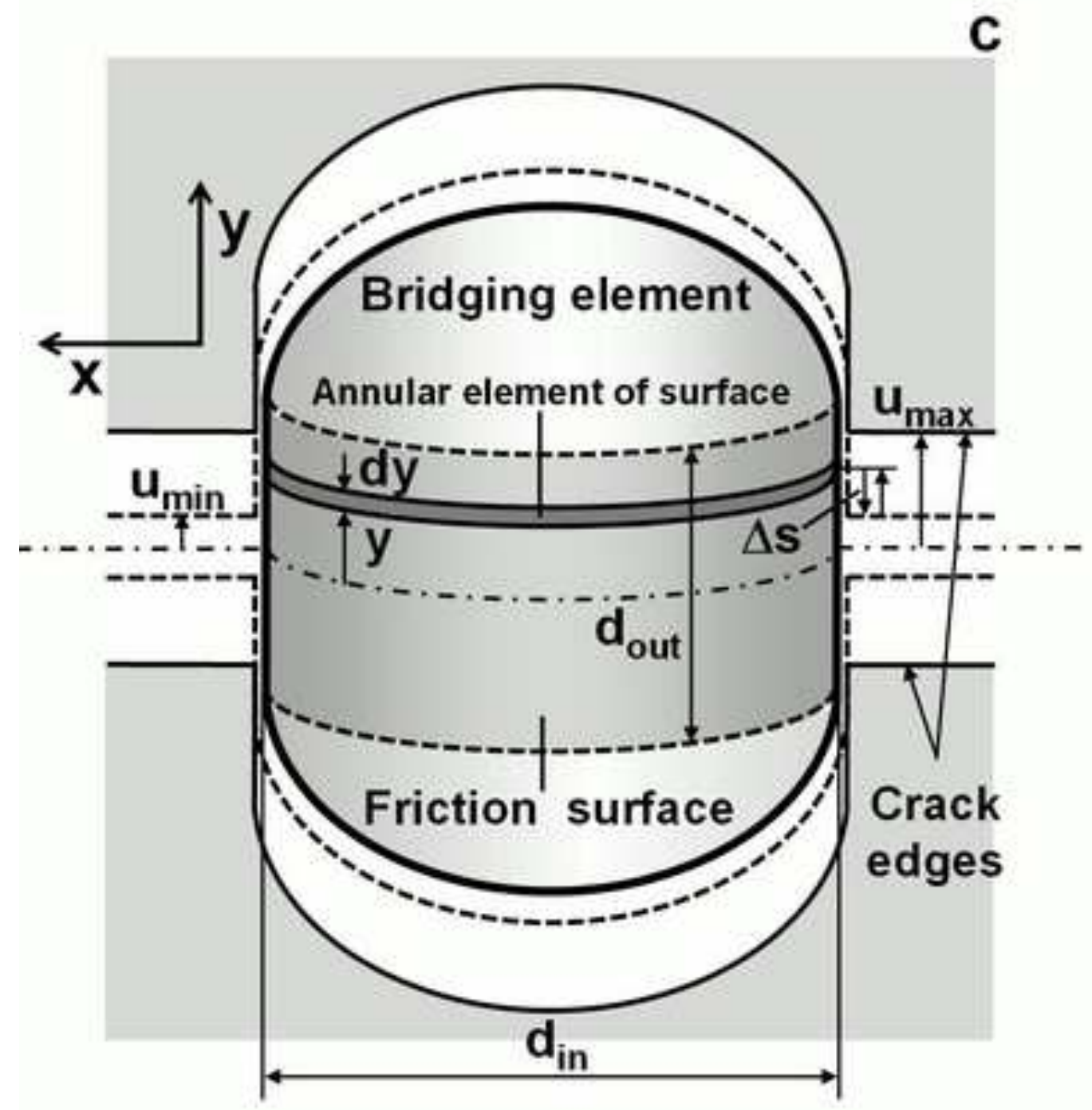




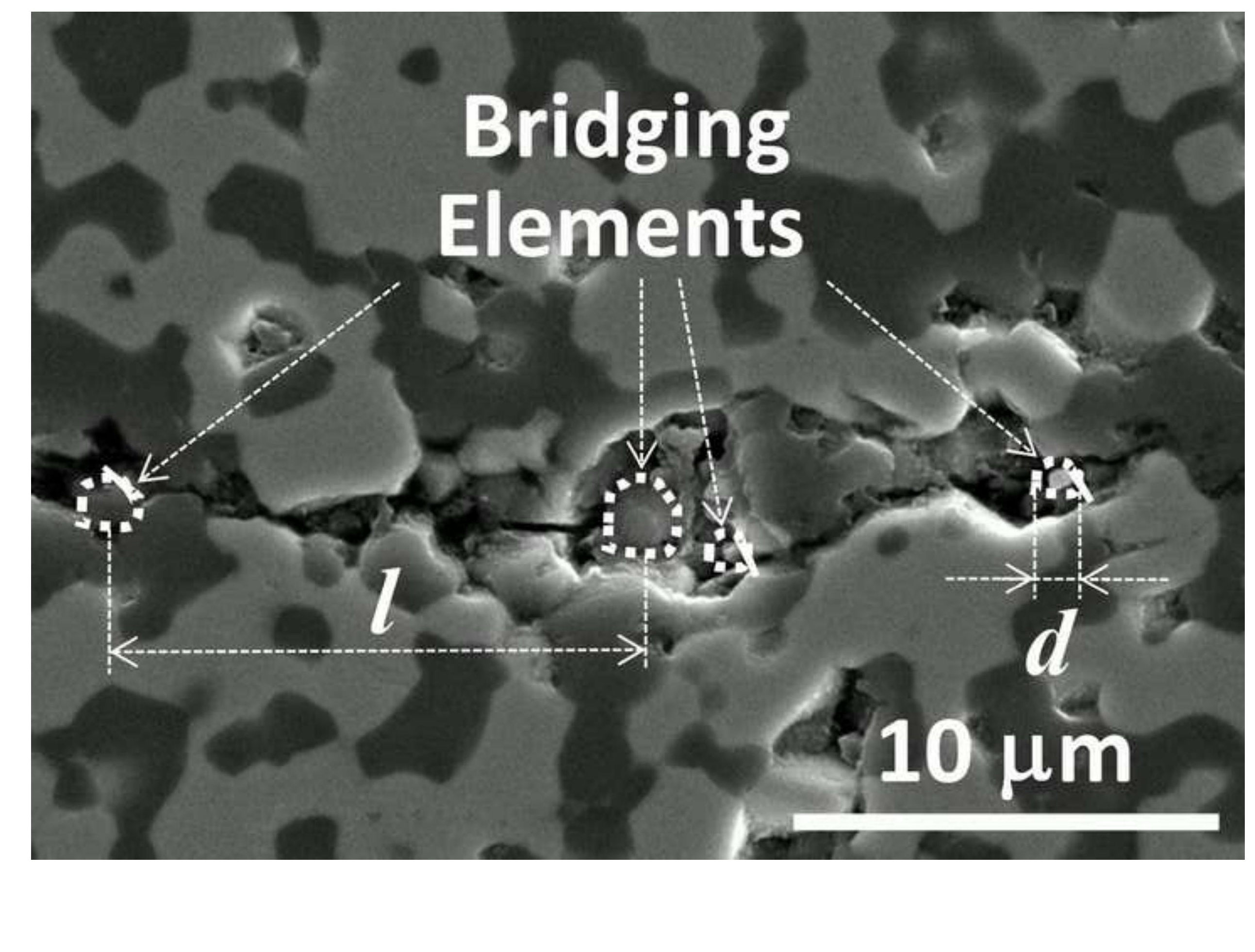

\section{Bridging Elements}

\section{.}
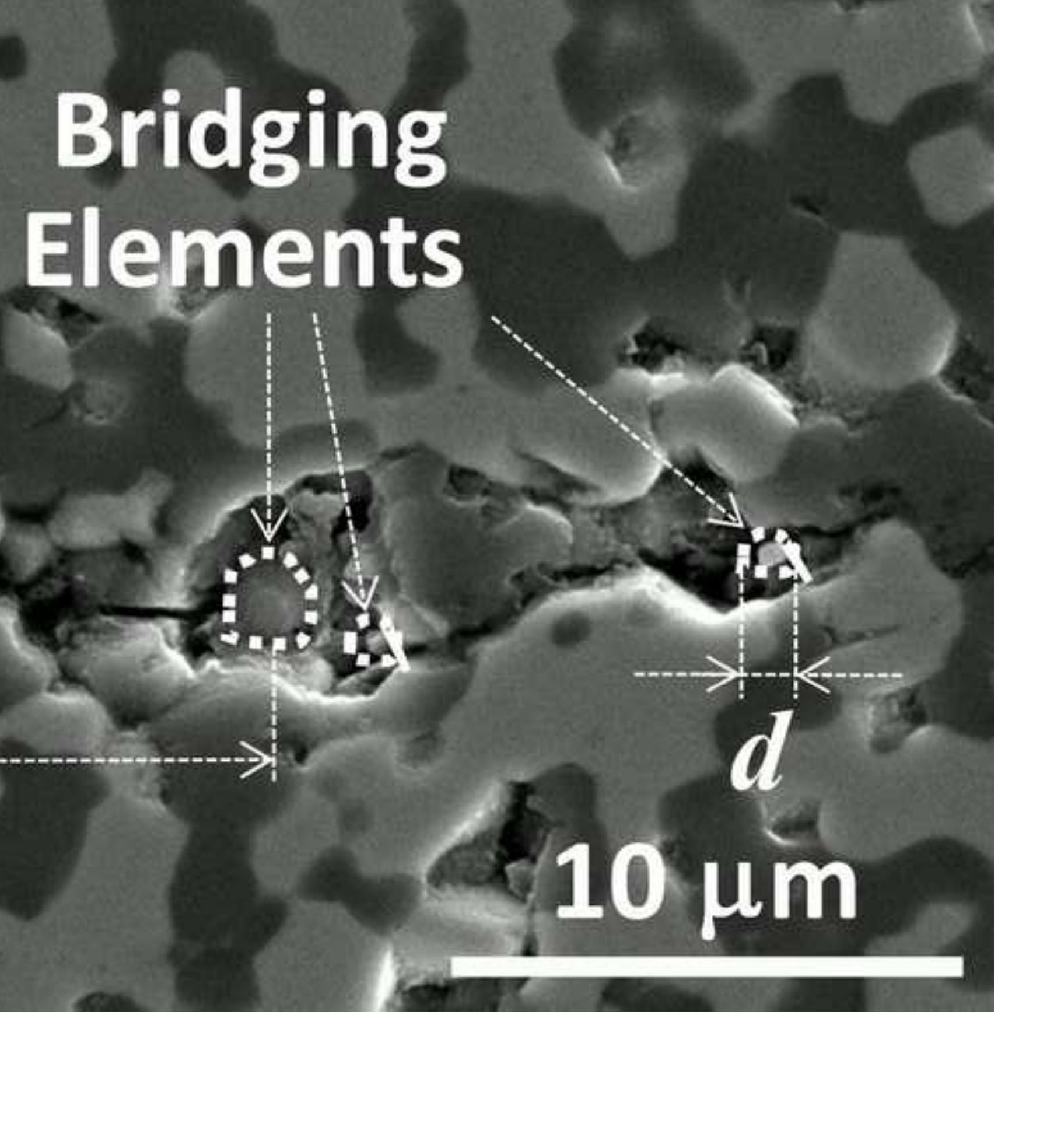


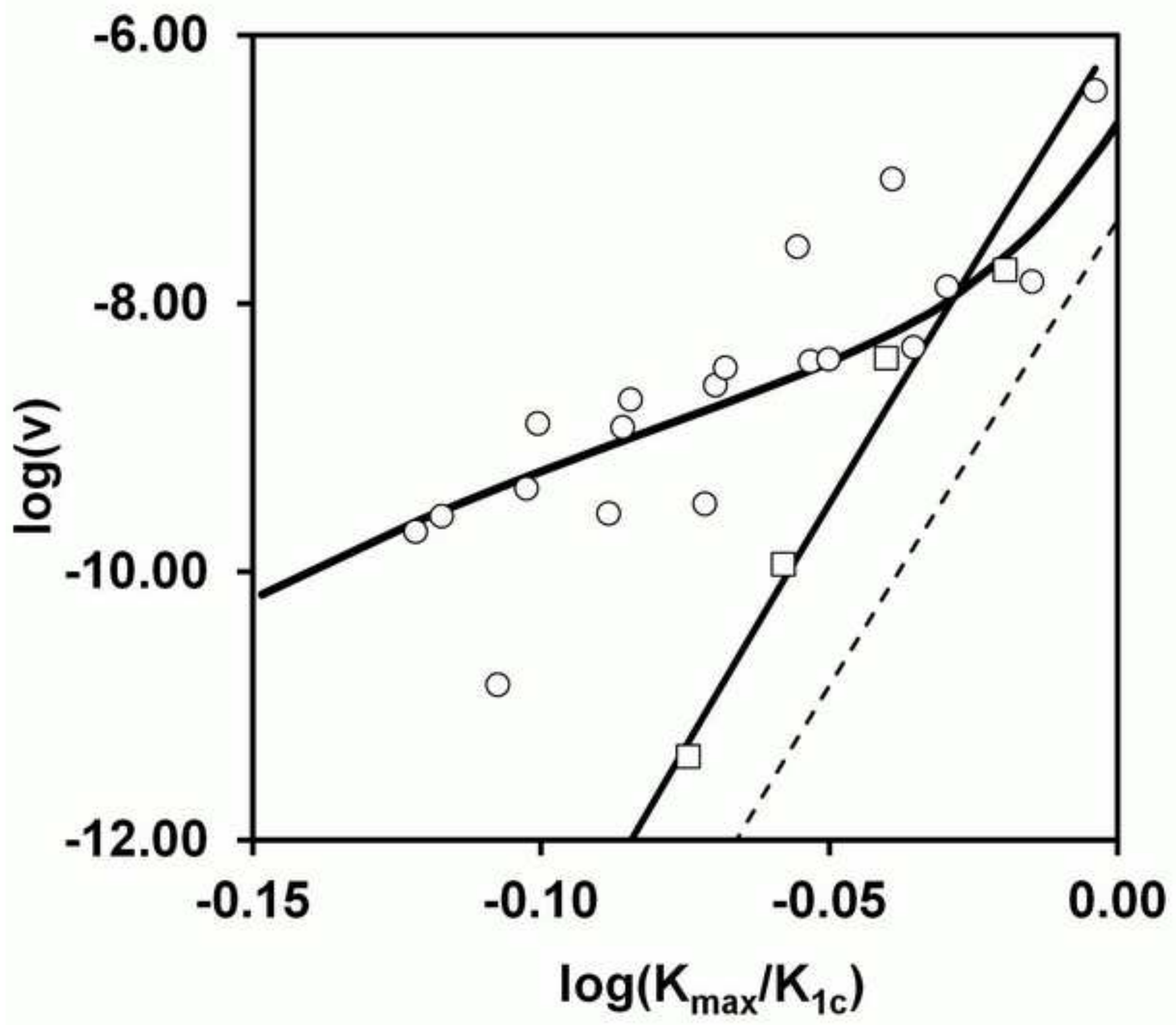



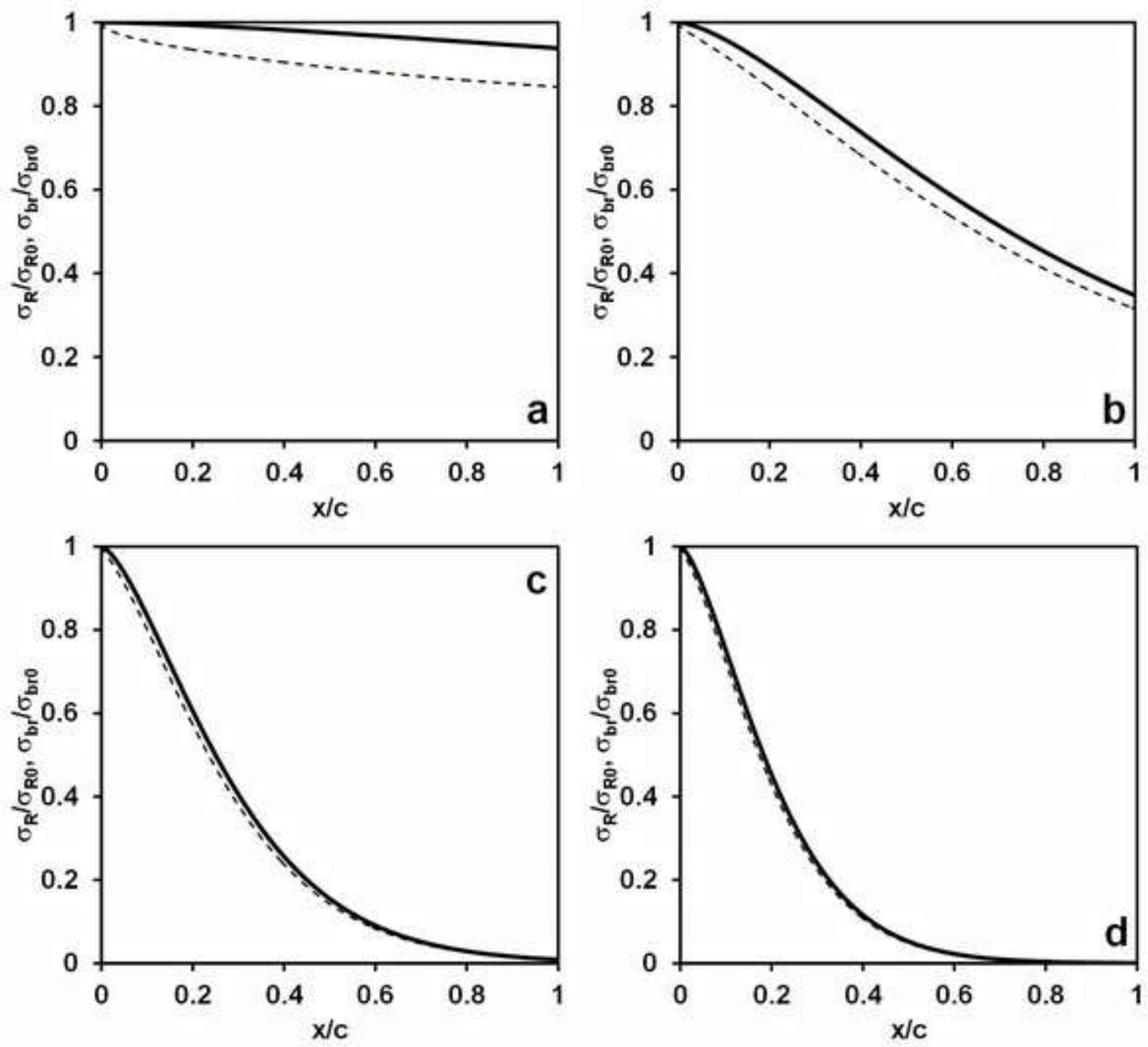


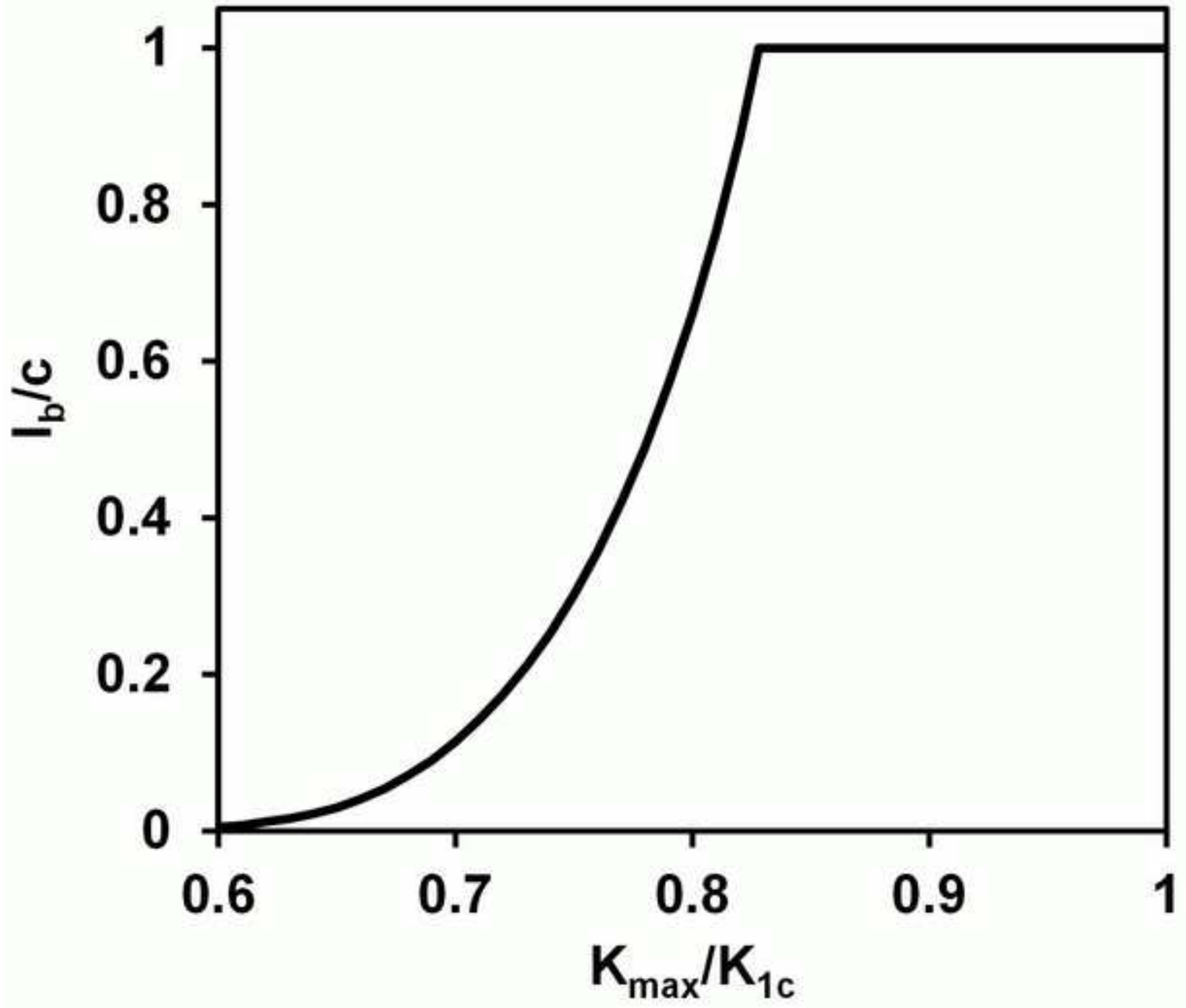




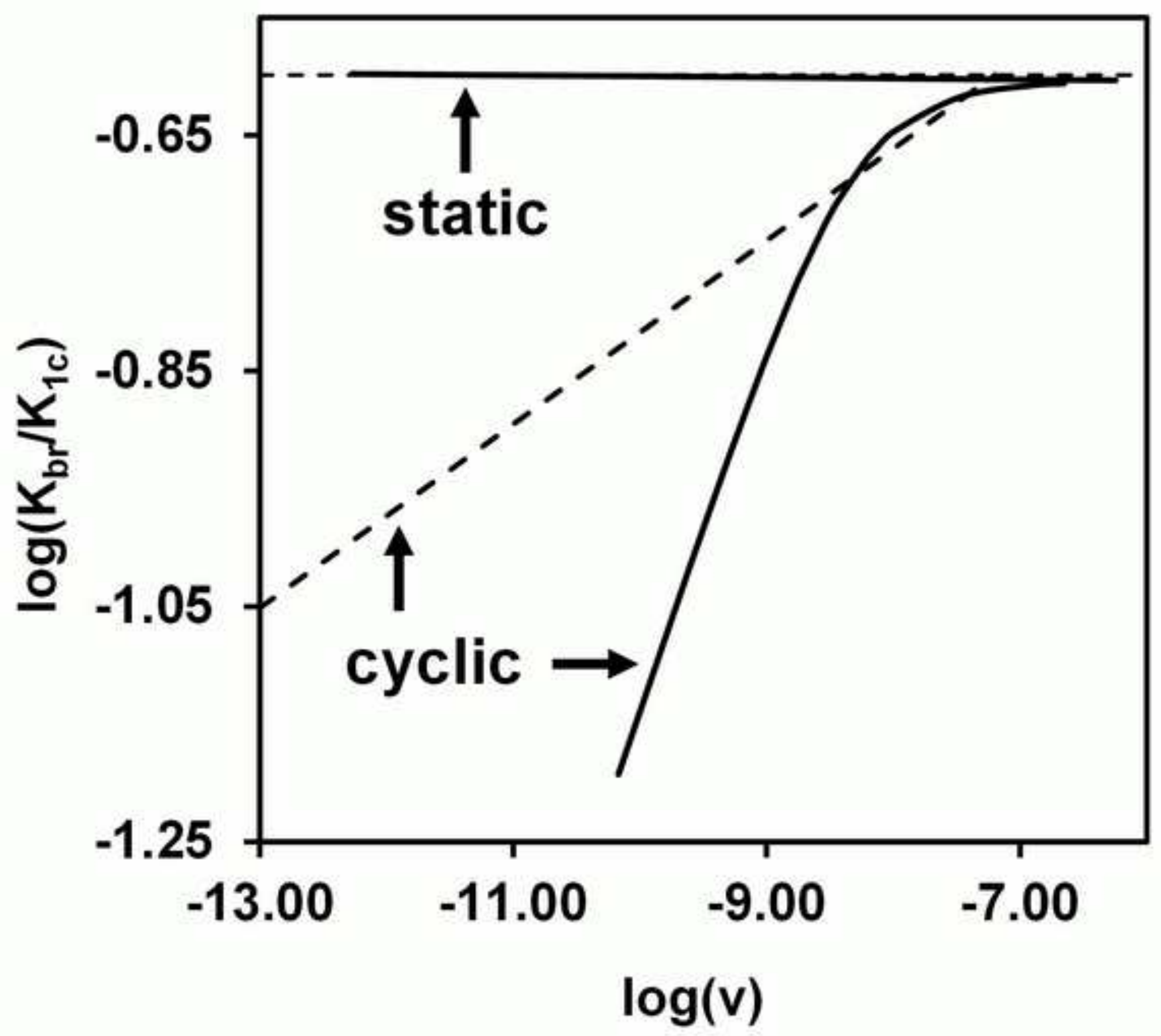

